\title{
Bottleneck for Rural Enterprises to Implement Intelligent Management and its Breakthrough
}

\author{
Shengjun Li \\ Xingtai University, Xingtai, 054001, China
}

Keywords: rural enterprises, intelligent management

\begin{abstract}
In the modern society, the enterprise mode is changing greatly, and the enterprise management has stepped toward the intelligent management, in some sense, the enterprise intelligent management is an inevitable trend for the enterprises to achieve self-perfection and update. In recent years, the rural reform in China has become a hot topic, and many medium and small enterprises have successively settled in rural areas, generating the intelligent management of rural enterprises. The exploration on relevant methods and modes of intelligence of rural enterprises is an important task. The modern rural enterprises mainly simulate the urban enterprises, but there is still a large difference between the rural enterprises and urban enterprises, so they cannot mentioned in the same breath. There exist many regional problems in the process of implementing the intelligent management of rural enterprises, but upon the unremitting efforts of many people, great breakthrough has still be achieved. In this paper, relevant topics on the rural enterprises' implementation of intelligent management are deeply discussed and analyzed.
\end{abstract}

\section{Introduction}

The informatization management in intelligent management is a vital part, and the internal competition of relevant enterprises is generated from it. China has a great plan, the Chinese Dream, and the realization of it is to achieve the rapid development of the national economy. China is a large population country, with current population of about 1.3 billion, in which the rural population accounts for 900 million. The top priority of developing the national economy is to make the farmers become rich, and the rapid development of modern rural enterprises has brought substantial contribution to the Chinese Dream, which cannot be underestimated. Therefore, the state increases support to reform the rural enterprises and make the operation mode intelligent and informationized. The interference of rural enterprises makes the rural economic system change greatly, transforming the rural economic pattern only relying on the agriculture into corporatization, and there also exists the deep processing of agricultural products in the corresponding enterprise cooperation scope, making the agricultural products form the pattern of local seeding local direct sales. The purpose of improving the farmers' profit for the construction of informationized construction of rural enterprises is to stabilize the market status of the rural enterprises and increase the competitiveness.

\section{Overview of enterprise intelligent management}

The primitive enterprises mainly carry out manual operation, with a low operation efficiency, and a lot of time and energies are wasted. In the continuous development, the automation is contacted correspondingly in the continuous development, and the advanced instruments and equipment form mechanical production, with outstanding contribution in the saving of labor. The modern information science and technology continuous develop and make progress, driving the mechanical production of enterprises and forming an integrated information pattern of production, sales and management, which does not only save a lot of labor, but also reduces the work amount of the technicians. The intelligent management system is mainly to combine the intelligent system to program relevant instructions intelligently and blend the whole system into various links of the enterprise production. For example, the modernized automobile automatic operation system inputs people's instruction to the corresponding level, and the system will automatically process relevant data for accurate judgment and operation, with the function of self-maintenance and repair in the 
intelligent system, and necessary update is available via the corresponding data. The intelligent market has an infinite development space, whether in application or technical improvement. In this paper, meticulous discussion and analysis are made aimed at the application of intelligent management system of the rural enterprises.

\section{Intelligent of rural enterprise intelligent management will become the inevitable trend}

At present, the enterprise competition is very fierce, only with sufficient advantages can the enterprises take the dominant role in the process of enterprise management and commercial operation, and in the core status in the process of commercial operation is the obtaining of commercial information and processing of core data. Some large enterprise equip the corresponding intelligent operation system to acquire the commercial information and analyze the commercial data, while within the rural enterprises, there is no reasonable and perfect operation concept, nor is there reliable and mature operating system, so the intelligent management of rural enterprises is the premise to guarantee the smooth operation and even the root. The establishment of rural enterprise intelligent management system solves a series of difficulties like imperfect commercial information within the enterprises, scattered data and waste of human resources within the enterprises. On the contrary, the intelligent management system can obtain the latest commercial information in the specific operation procedures and unique open platform, and after obtaining the information resources, the system will reasonably classify and integrate the commercial information and data, making it convenient for relevant staff and managers to obtain the data clearly and have an effective comparison, and on this basis, the problem of inputting a lot of human resources is solved. Ultimately, the commercial competition is the competition for technology and management system, therefore, it is quite necessary to implement an effective intelligent management system in a rural enterprise.

\section{Analysis on development status of modern rural enterprises}

The economic development speed ranks the first in the world, but to achieve the comprehensive management of the national economy, relevant national leaders make great effort to reform the rural economic system and support and encourage the small and medium-sized enterprises to settle in the rural areas in system, so the rural economy is initially relieved. But the current situation is still serious, mainly reflected in the fact that the small and medium enterprises face the difficulty of backward operation and management system, and in comparison with the intelligent relation of the large enterprises, the rural enterprises have no strength to resist at all, mainly because the rural enterprises do not have enough economic strength and enough advantages in environment. So they cannot keep pace with the times in equipment and do not have corresponding advantages in recruitment, the enterprises do not have competitiveness without the support of core technology, and there will be no corresponding technical development and improvement without talents, in such way, the vicious circle forms the current status of the backward rural enterprise management.

\section{Problems in implementation of intelligent management for rural enterprises}

\section{Lack of commercial information}

With the increasing development of science and technology, it is more convenient to obtain information, but in many rural areas, science and technology are not developed enough, and it is difficult to obtain information. In this situation, enterprises mainly depend on the experience accumulated for a long time, and then combine it with the specific practices, so as to establish the management policies conforming to the development of rural enterprises. With the continuous development of science and technology, the information technology has also been developed rapidly. The development of enterprises depends on a lot or information and data, while the lack of modern scientific and technological knowledge has become a main obstacle for the rural enterprises to implement the intelligent management. IBM once counted that there is a great utilization of data 
globally everyday, which means that the rural enterprises establish decisions without relying on the experience any longer, but obtain the information through data analysis, so as to provide help for the decision-making, which increases a lot of difficulties for the enterprises' decision-making, and is also another important problem for the rural enterprises to implement intelligent management. In front of a lot of data, if it is able to sort the most needed information within the shortest time, it is able to improve the enterprises' efficiency, so as to increase the competitiveness.

Too huge information and data statistical work

After the rural enterprises enter the intelligent management stage, the corresponding data are not the difficulty, because the research and development of information collection with intelligent system is underway smoothly. Facing the data resources with a huge information volume, it sill cost nearly 1 day and a half to complete the data and data processing manually. In front of such difficult information analysis and data statistics, the management personnel of relevant rural enterprises can only make improvement in the operating system, invite relevant professional technicians for comprehensive detection and purchase the intelligent business information processing system.

Too high business cost and lack of talents

The rural investment enterprises are mainly medium and small enterprises, with not powerful comprehensive strength and it is not only needed to comprehensively plan, but also input a lot of funds, as well as appoint relevant professional talents in the process of intelligent management, and without reliable economic support, it is unable to purchase the advanced intelligent operation equipment, and if there are no relevant professional talents, it will be more difficult for the rural enterprises to implement intelligent management.

The intelligent enterprise management system contains high science and technology, the non-professionals cannot carryout the operation, repair and maintenance and even will be more difficult to compile the corresponding intelligent programs without the corresponding theoretical learning. The professional talents have a higher standard for job selection, with expected work environment basically some developed cities, so the corresponding rural enterprises often do not have the geographical advantages. If there is a lack of professional talents, it will be impossible to develop the intelligent management stably.

\section{Progress of intelligent management of rural enterprises}

Although there are many difficulties met in the intelligent management of rural enterprises, the overall intelligent direction of enterprises cannot be changed. In the modern enterprise development situation, intelligent management system is the evitable trend, being the real core technology. Therefore, relevant technical and management personnel have input a lot of energies, and breakthrough progress has been made.

Information obtaining and data construction

The obtaining of business information is the fundamental for the enterprises' normal operation. If an enterprise loses the approach to obtain the business information, it will face the risks of bankruptcy, the intelligent management system of rural enterprises brings unprecedented power to the development of the rural enterprises, and there are many approaches for the intelligent system to obtain the business information, first, the intelligent management system owns a broad information collection platform, which will actively relevant valuable information in the broad information platform, and update the relevant information automatically, additionally, the intelligent management system also owns huge data processing function, relevant staffs of the rural enterprises carry out a lot of development and research, fully utilize the intelligent enterprise management system in the data processing, clarify the complicated data and compare the data automatically to summarize the corresponding conclusion for reference and decision-making by the enterprise managers.

Reasonable classification and processing of intelligent system to data

The difficulties met in the intelligent management process of rural enterprises are not only the information collection, more importantly, to reasonably classify the information collected and carry out relevant data statistical work, and these difficulties are met and solved in the implementation of 
intelligent management. In the process of constructing the intelligent management system, the rural enterprises add data warehouse technology research and development, and maturely develops and acts with this technology maturely, not only the information is comprehensive, but the information and data excels will also be generated automatically to reasonably decompose the information collected, compare and summarize the relevant data, and provide meticulous analysis and calculation for the senior managers within the rural enterprises in the form of portal. The advanced intelligent information processing system can save and timely update data resources for the rural enterprises, count the latest data for professional comparison, so as to make it convenient for relevant responsible personnel to make a reasonable judgment and selection by depending on the data. This solves the difficulty of difficult classified summary, time and labor consumption brought by the huge information volume to a certain extent.

Compression of business cost

Any enterprise is for profit, so the business cost is of vital importance, and the rural enterprises are of no exception. The e-commerce becomes the favorite of the enterprises because of its convenience, rapidness and low price. There will be market only after these two characteristics are met. In recent years, the international trade has been developed rapidly, but the support of e-commerce is also inseparable. The application of e-commerce in the international trade significantly reduces the trade cost, including the transaction cost, purchase cost and transportation cost. The e-commerce mainly conveys and exchanges information with computer network as the media, in this way, the information transmission is more convenient and the efficiency is improved significantly. In order to ensure the characteristics of convenience, rapidness and low price of the e-commerce, first it is needed to comprehensively understand the cost composition of the e-commerce, so as to control the cost from various links, minimize the cost, and then, it is required to improve the efficiency of e-commerce. But actually, the control toe the cost of e-commerce is far from being satisfactory, the cost of e-commerce has always been exceeding people's expectation, and even compared with the traditional commerce, the e-commerce has higher cost. In the times of legal governance, the development of any thing should be regulated by the laws, so is the e-commerce. As the saying goes, nothing can be accomplished without norms or standards, establishing some rules can reduce some unnecessary troubles of course, but brings obstacles to the operation of-commerce, and the cost also increases. Therefore, for the decrease of commercial cost, legal restriction is also an obstacle. In addition, the development of e-commerce is not mature enough, with some risks in operation, which are uncertain and will cause some implicit cost, therefore, to reduce the business cost, the operation risks must be considered.

In consideration of lack of talents in the rural enterprises, it is required to actively coordinate with the corresponding universities, cultivate relevant targeted talents in the universities, with their tuition fees borne by the relevant enterprises, and meanwhile, issue the living subsidy regularly and after graduation, the graduates enter relevant rural enterprises to practice and to be familiar with the work environment. Besides, establish the corresponding award and bonus in the research and development of relevant technicians for encouragement, so as to mobilize the incentives of the staff.

\section{Conclusion}

The intelligent management of rural enterprises is the key to achieve the breakthrough progress of the Chinese national economy, so it is widely concerned. First, relying on the great support of the state, it is an extremely complex and long-term project to establish the intelligent management system of rural enterprises, which needs people's unremitting efforts and countless realistic problems will be met, including failure to collect all business information, too heavy data classification and summary task for relevant information, waste of a lot of energies of the enterprises and work staff, increase of business cost, urgent fund circulation and lack of processional talents etc. Aimed at the corresponding problems, it has become a joint striving objective to carry out necessary intelligent reform of rural enterprises, and meanwhile, through a series of problem solutions like technical research and development and coordinative cultivation of university talents have made the intelligence of rural enterprises have initial scales. The 
establishment of rural enterprise intelligent management needs the joint efforts of all villagers, and the final purpose to creating intelligent enterprises is to create better revenues and incomes for the villagers, so the villagers should actively cooperate with relevant regulations and systems to improve their overall quality, so as to lay a solid foundation to really realize the intelligent villages.

\section{References}

[1] Chen Qishen, Know Theory, Know Oneself, Know Others, Know Application, Regulated Process to Successfully Implement ERP [M] Beijing: Electronic Industry Press, 2007: 266

[2] Wang Hongshui, Strengthen Enterprise Productivity with Informatization, Create More Benefit for Enterprises [J] Metallurgical Automation , 2006, 31 (01):1-5

[3] Ji Xiaoyuan, Single and Intelligent Methods and their Application in Cast Informatization [D]. Wuhan: Huazhong University of Science and Technology

[4] Seng J, Kong I L. A schema and ontology-aided intelligent information integration[J]. Expert Systems with Applications.

2009, 36(7): 10538-10550.

[5] Yang S, Chang Y. An active and intelligent network management system with ontology-based and multi-agent techniques[J]. Expert Systems with Applications. 2011, 38(8): 10320-10342.

[6] Tong Yan, Primary Discussion on Informatization Construction of Township Enterprises, [J] Township Enterprises, 2004 (04)

[7] Gao Zhengxian, Li Zhongxue, Role-based Access Control Strategy under Web Environment and its Realization [J] Computer Engineering, 2004, 30 (8) 